\title{
Stratified shear flow: experiments in an inclined duct
}

\author{
COLIN R. MEYER $\dagger$ AND P. F. LINDEN
}

Department of Applied Mathematics and Theoretical Physics, Centre for Mathematical Sciences, University of Cambridge, Wilberforce Road, Cambridge, CB3 0WA, UK.

$$
\text { (Received -; revised -; accepted -.) }
$$

We present results of experiments on stratified shear flow in an inclined duct. The duct connects two reservoirs of fluid with different densities, and contains a counterflow with a dense layer flowing beneath a less-dense layer moving in the opposite direction. We identify four flow states in this experiment, depending on the fractional density differences, characterised by the dimensionless Atwood number, and the angle of inclination $\theta$, which is defined to be positive (negative) when the along-duct component of gravity reinforces (opposes) the buoyancy-induced pressure differences across the ends of the duct. For sufficiently negative angles and small fractional density differences the flow is observed to be laminar ( $L$ state) with an undisturbed density interface separating the two layers. For positive angles and/or high fractional density differences three other states are observed. For small angles of inclination the flow is wave-dominated and exhibits Holmboe modes ( $\mathrm{H}$ state) on the interface with characteristic cusp-like wave breaking. At the highest positive angles and density differences there is a turbulent ( $T$ state) high-dissipation interfacial region typically containing Kelvin-Helmholtz $(\mathrm{KH})$-like structures sheared in the direction of the mean shear and connecting both layers. For intermediate angles and density differences an intermittent state (I state) is found, which exhibits a rich range of spatio-temporal behaviour and an interfacial region that contains features of KH-like structures and of the other two lower-dissipation states: thin interfaces and Holmboe-like structures. We map the state diagram of these flows in the Atwood number $-\theta$ plane and examine the force balances that determine each of these states. We find that the $\mathrm{L}$ and $\mathrm{H}$ states are hydraulically controlled at the ends of the duct and the flow is determined by the pressure difference associated with the density difference between the reservoirs. As the inclination increases, the along-slope component of the buoyancy force becomes more significant and the I and $\mathrm{T}$ states are associated with increasing dissipation within the duct. We replot the state-space in the Grashof number $-\theta$ phase plane and find the transition to the T-state is governed by a critical Grashof number. We find that the corresponding buoyancy Reynolds number of the transition to the T-state is of order 100, and that this state is also found to be hydraulically controlled at the ends of the duct. In this state the dissipation balances the force associated with the along-slope component of buoyancy and the counterflow has a critical composite Froude number.

\section{Key words:}

\section{Introduction}

Stratified turbulence is a fundamental problem in fluid mechanics with important implications across a wide diversity of applications from mixing in the oceans and atmosphere down to scales

$\dagger$ Email address for correspondence: colinrmeyer@gmail.com. 
associated with industrial processes. The interplay of buoyancy forces and turbulence is fascinating, and has been the subject of experimental, computational and theoretical studies for over a century. Nevertheless, our understanding of the dynamical processes involved is far from complete, and this lack of understanding is reflected, for example, in our inability to predict mixing rates from the mean velocity and density fields.

Stratified shear flow is a canonical flow that can range from laminar to turbulent, and this paper describes novel experiments on stratified counterflow in an inclined square duct. The duct is connected to large reservoirs at each end, containing fluids with different densities. In the case of a horizontal duct the pressure differences at the two ends of the duct drives a counterflow with the dense fluid moving along the bottom of the duct and the light fluid moving along the top. This configuration has been studied by Lawrence and his group (Lawrence et al. 1991; Gu \& Lawrence 2005; Tedford et al. 2009), particularly in the context of the generation of Holmboe instabilities.

Stratified shear flow generated in a closed tilted channel has been studied in a series of influential papers by Thorpe $(1968,1971,1973)$. Thorpe's original experiment was inspired by a study of two immiscible fluids, water and carbon-bisulphide, in a tilted tube by Reynolds (1883). In Thorpe's experiments a miscible two-layer (or continuously stratified) fluid initially at rest in a horizontal channel is set into motion by tilting the channel. The component of gravity acting along the length of the channel produces a constant acceleration of the dense fluid towards the lower end of the channel and the light fluid towards the upper end. These beautiful experiments showed that once a critical velocity difference between the accelerating layers was reached, Kelvin-Helmholtz instabilities appeared and transferred mass and momentum between the counter-flowing layers.

The present experiment can be considered as a continuous version of the transient tilted channel experiment. Fluid entering from the dense reservoir is accelerated (or decelerated) by the component of gravity along the length of the duct, while the light fluid entering from the other reservoir is accelerated (or decelerated) in the other direction. Similar buoyancy-driven counterflow experiments in a sloping duct joining two reservoirs were studied by Kiel (1991). He considered ducts of square cross section but with a maximum length to height aspect ratio of 8 , significantly smaller than the ducts used in this present study. Most of the measurements were at angles considerably larger than those discussed in this paper although he presents shadowgraph and dye images for inclination angles $\theta=0$ and $2.5^{\circ}$. These images are consistent with those we discuss below. Tedford et al. (2011) carried out experiments where they temporarily tilted a duct containing a two-layer exchange flow, and measured the acceleration of the flow and the subsequent structure of the interface when the duct was returned to the horizontal. They also observed some features consistent with the experiments reported here.

We observe a wide range of flow phenomena depending on the angle of inclination and the density difference between the two reservoirs. The goal of this paper is to document the state diagram of these flows, and to determine the dynamical balances in each of the observed states. The paper is organised as follows. In $\S 2$, we describe the experiments and present examples of the flow states. In $\S 3$ we present the regime diagram for the flows and some quantitative measurements. In $\S 4$ we discuss the dynamical balances associated with the flow states, and the conclusions are given in $\S 5$.

\section{Experiments}

The experiments were conducted in a $L=3 \mathrm{~m}$ long duct with a square cross section $H=$ $0.1 \mathrm{~m}$ in height. The duct passed through a vertical barrier, which divided a large tank measuring $9.6 \mathrm{~m}$ long by $0.25 \mathrm{~m}$ wide by $0.5 \mathrm{~m}$ deep into two halves. These two halves were filled with salt solutions of different densities $\rho$ and $\rho+\Delta \rho$. The barrier had a central portion made of flexible 


\section{Higher Density Lower Density}

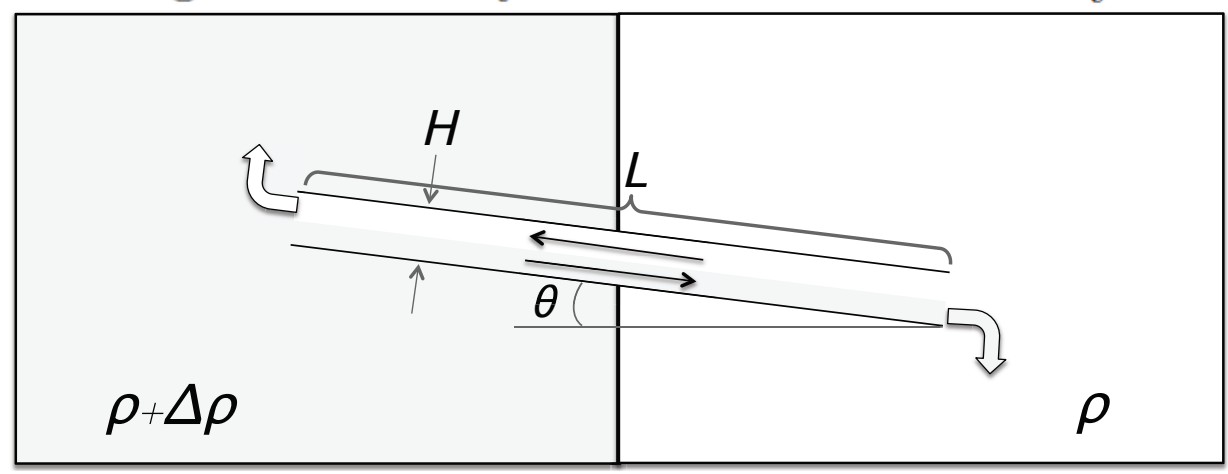

FIGURE 1. A schematic of the experimental set-up (not to scale), shown in the configuration with $\Delta \rho>0, \theta>0$ so that the flow in each layer is accelerated by the along-slope component of gravity.

rubber sheet which allowed the duct to be inclined at an angle $\theta$ to the horizontal. We denote $\theta>0$ when the upper end of the duct is in the denser reservoir $(\Delta \rho>0)$ : in that case the dense fluid is accelerated down the duct and the lighter fluid is accelerated up the duct, by the component of gravity along the length of the duct. When $\theta<0$ the counterflow is decelerated by this component of gravity (figure 1).

Initially, the duct was open to the denser side and capped on the other end, and the fluid was at rest. The depths of the water in the two reservoirs were adjusted so that the hydrostatic pressures at the depth of the mid plane of the duct were equal. This ensured that the net barotropic flow through the duct was minimised. In practice there was some initial net barotopic flow but the subsequent change in reservoir levels led to a flow with no net volume flux over the majority of the experiment. As discussed in $\S 3.2$, experiments in which measurements of the mass flux of salt indicated a significant barotopic flow were not used to estimate the net exchange between the two reservoirs.

Each experiment was started by removing the cap from the end of the duct and was terminated before the inflow into the duct was affected by the fluid that had previously entered the reservoirs through the duct. Typically, it was possible to run an experiment for several minutes before any observed change in the inflow conditions occurred, which was much longer than the typical transit time (30s) through the duct. With each run the density difference between the reservoirs decreased due to the transfer of salt through the duct. After an experiment the end cap was replaced to seal the duct and both sides of the tank were completely mixed using submersible pumps, and the densities measured to obtain an estimate of the time-averaged mass flux through the duct. The density was measured by withdrawing samples and measuring them in an Anton Paar density meter, which gave values accurate to $10^{-2} \mathrm{~kg} \mathrm{~m}^{-3}$.

A total of 255 experiments were run, including 85 with half the duct $(L=1.5 \mathrm{~m})$, with density differences from $2 \leqslant \Delta \rho \leqslant 200 \mathrm{~kg} \mathrm{~m}^{-3}$, i.e. $1000 \lesssim \rho \lesssim 1200 \mathrm{~kg} \mathrm{~m}^{-3}$. Over this range of densities the average kinematic viscosity $\nu$ of the salt solutions varied between $1.01-1.19 .10^{-6}$ $\mathrm{m}^{2} \mathrm{~s}^{-1}$ and the molecular diffusivity $\kappa$ of salt in water varied between $1.26-1.55 .10^{-9} \mathrm{~m}^{2}$ $\mathrm{s}^{-1}$. The Schmidt number $S c=\nu / \kappa \sim 10^{3}$ is large and so the effects of molecular diffusion of salt are negligible. The geometry of the experiment constrained the inclination to $|\theta| \leqslant 4^{\circ}$ and experiments were run over the range $-1^{\circ} \leqslant \theta \leqslant 4^{\circ}$. Qualitative observations of the flow were made with shadowgraph. A section of the duct, measuring $0.3 \mathrm{~m}$ in length and located approximately $1 \mathrm{~m}$ from one end of the duct, was illuminated by parallel light and the image projected onto a translucent screen. The flow as also observed at other locations and this section 


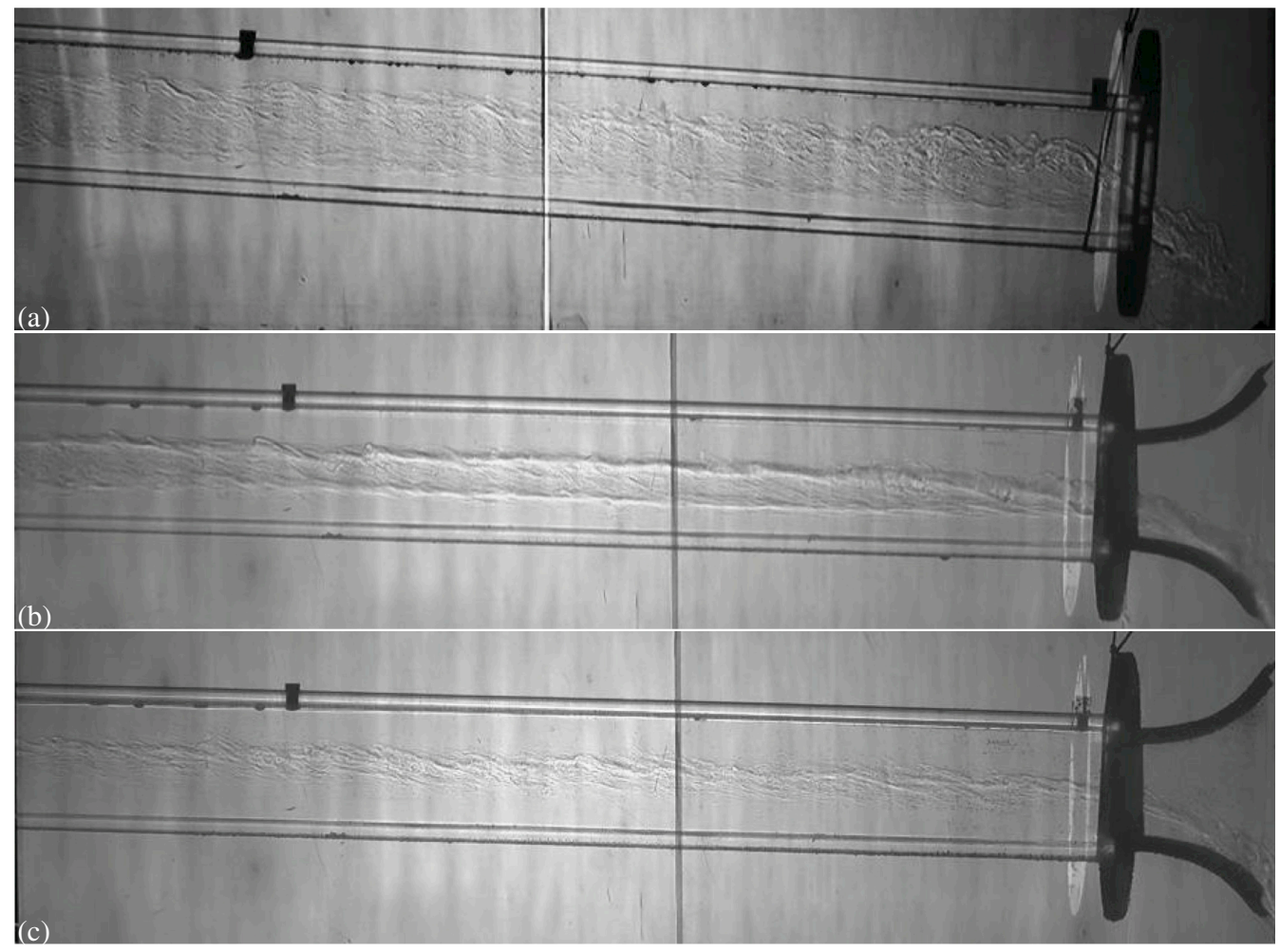

FIGURE 2. Shadowgraph images over $1 \mathrm{~m}$ of the duct including the lower end. The effect of the entry diminishes rapidly and a consistent dynamic interface is established. The flow states are (a) $\mathrm{T}$ state with $A=3.9 \times 10^{-3}, \theta=3.5^{\circ}$; (b) I state with $A=8.3 \times 10^{-3}, \theta=1.5^{\circ}$; and (c) I state with $A=6.7 \times 10^{-3}$, $\theta=1.5^{\circ}$

was found to be representative of the flow away from the entry regions which extended about $0.2 \mathrm{~m}$ into the duct from each end.

\section{Results}

\subsection{Qualitative Results}

On removing the end cap a gravity current of light fluid developed. After the nose of the gravity current exited the duct, the flow accelerated and established a counterflow with the dense fluid moving towards the light reservoir underneath the light fluid moving in the opposite direction. Figure 2 shows three shadowgraph images of the flow over a length $1 \mathrm{~m}$ of the duct including one end. In one case the end is abrupt, while in the other two cases smooth entry and exit shapes are added to the upper and lower boundaries. We see no effects of adding these smooth entry and exit regions compared with the abrupt end shown in figure 2a. Furthermore, we observe that the flow is rapidly established and the effects of the entry and exits of the two layers extends less than two duct heights. Consequently, the observation window referred to in $\S 2$ provides images representative of the flow along the majority of the duct. These images also show that the exiting lower layer accelerates and decreases in depth near the end of the duct before flowing into the reservoir. Although we do not have velocity measurements to confirm this, the exiting layer appears to become supercritical as it leaves the duct. We discuss further aspects of the hydraulic nature of the flow in $\S 4$.

We observed the four different flow states $(\mathrm{L}, \mathrm{H}, \mathrm{I}$ and $\mathrm{T})$ shown in figure 3 . For $\theta<0$ and low 

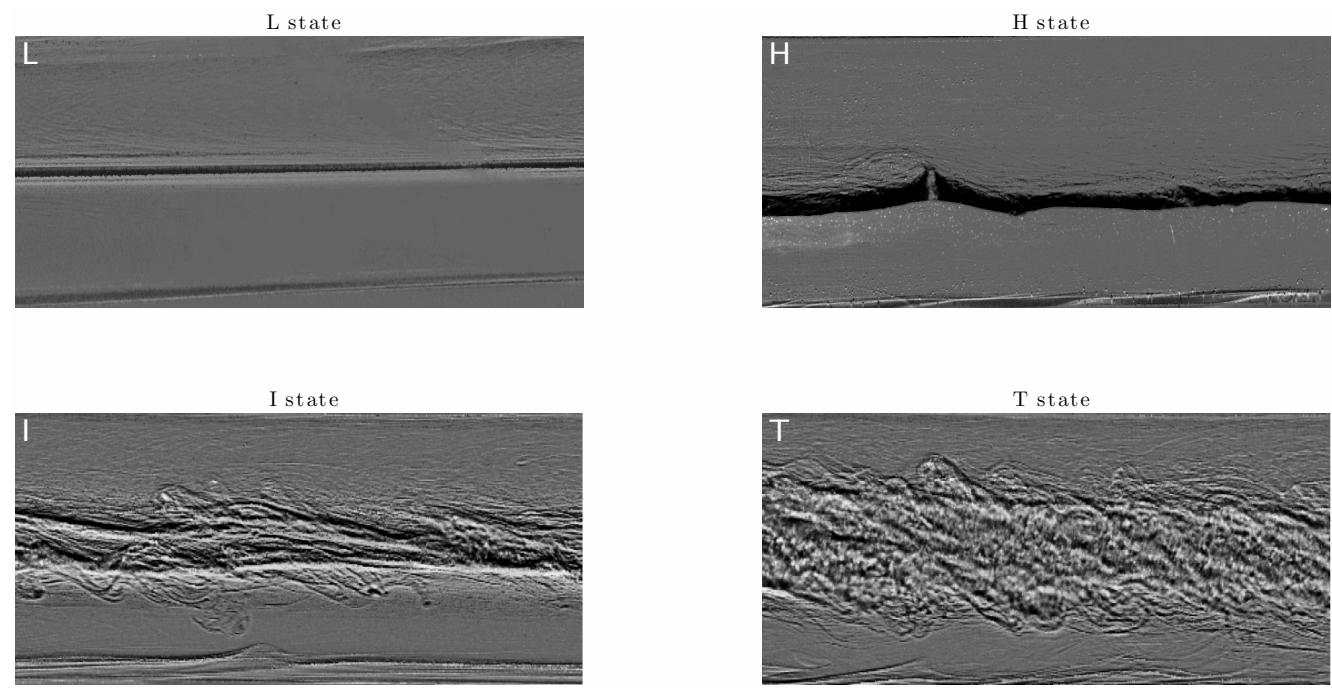

FIGURE 3. Shadowgraph images of the four primary flow states. Each panel image is taken approximately $1 \mathrm{~m}$ from the right hand end of the duct, and shows the entire depth $(10 \mathrm{~cm})$ and has horizontal extent $22 \mathrm{~cm}$. The angle and Atwood number for each case are $\mathrm{L}: \theta=-1^{\circ}, A=6.7 \times 10^{-2}$, $\mathrm{H}: \theta=0^{\circ}, A=2.0 \times 10^{-2}, \mathrm{I}: \theta=1.5^{\circ}, A=4.6 \times 10^{-3}$, and $\mathrm{T}: \theta=1.5^{\circ}, A=4.6 \times 10^{-3}$. The camera is aligned with the duct. The structures visible near the lower boundary are a combination of optical reflections of the interfacial structures which do not affect the flow dynamics and interactions between eddies originating at the interface and the wall.

values of $\Delta \rho$ the flow is in the laminar $\mathrm{L}$ state (figure $3 \mathrm{~L}$ ) in which the interface is undisturbed, with no vertical motion greater than the thickness of the interface $(2 \mathrm{~mm})$ and with no apparent mixing between the two layers. It is possible for sufficiently high Reynolds numbers that the flow in the individual layers is turbulent, although at the velocities achieved in these experiments there is little evidence of this. However, we define the state in terms of the deformations of the interface as described above. As $\theta$ and/or $\Delta \rho$ increases the flow exhibits the other three flow states shown in figure 3: $\mathrm{H}, \mathrm{I}$ or $\mathrm{T}$. We will now describe each flow state in turn.

The $\mathrm{H}$ state, figure $3 \mathrm{H}$, shows the characteristic cusped structure observed in previous experiments in horizontal channels (Lawrence et al. 1991; Tedford et al. 2009). This state was observed for small angles $\left(\theta \leqslant 1^{\circ}\right)$ and for smaller values of $\Delta \rho$, and exhibited cusped waves travelling in opposite directions on the two sides of the interface, with, in most cases, intermittent breaking at the cusps. This structure is characteristic of the Holmboe mode of interfacial instability when the thickness of the interfacial shear is larger than the extent of the density gradient (Holmboe 1962; Lawrence et al. 1991; Caulfield 1994). At the largest values of $\theta$ and $\Delta \rho$, the T state flow was characterised by an interfacial region in which large amplitude $\mathrm{KH}$-like structures were seen (figure 3T). These structures were tilted in the direction of the mean shear and appeared to stretch from one layer to the other, like KH billows that have undergone a secondary three-dimensional instability, providing a direct connection between the two layers and provide the possibility of significant mass and momentum fluxes between the two layers in this state. In each of the L, H and $T$ states the flow remains statistically steady.

The fourth I state, however, was not steady but instead exhibited strong spatio-temporal intermittency (figures $3 \mathrm{l}$ and 4). This state occurred as an intermediate state between the $\mathrm{H}$ and $\mathrm{T}$, and contained a range of different flows with characteristics of both. The interfacial region contained a mixture of thin interfaces, cusp-like modes, sheared KH-like modes and small scale structures all in varying amounts which changed with position along the duct and with time during an experiment. Transitions were observed from an L-like or $\mathrm{H}$-like state to a more $\mathrm{T}$-like state and 

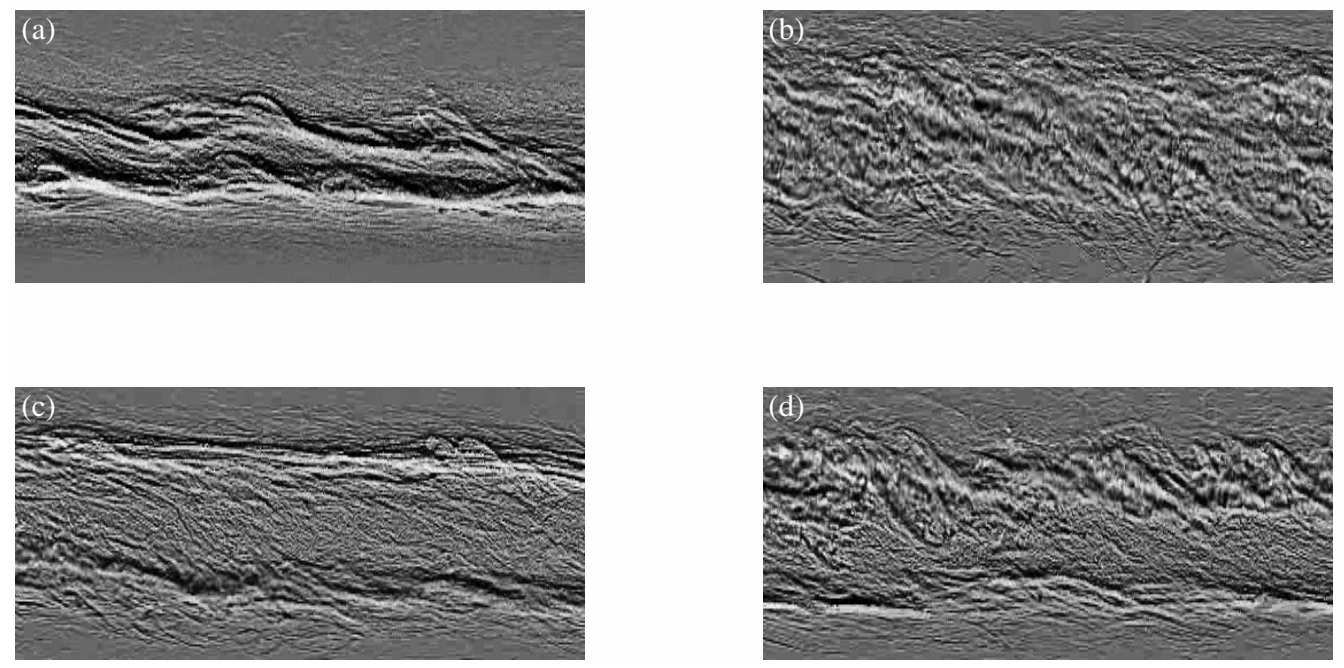

Figure 4. Different flows observed at different times $t$ in I state $\left(\theta=1.5^{\circ}, A=4.6 \times 10^{-3}\right)$ : (a) quasi-laminar flow with breaking internal waves and other layered structures ( $t=70 \mathrm{~s}$ ), (b) KH-like turbulence $(t=90 \mathrm{~s})$, (c) multi-layer quasi-laminar flow $(t=120 \mathrm{~s})$, and (d) multi-layer turbulence $(t=150$ $\mathrm{s})$. Each panel is $10 \mathrm{~cm}$ high by $22 \mathrm{~cm}$ long.

back again, and different states were observed along the duct at the same time. This rich zoo of flow structures can be seen in the movie in the supplementary material.

\subsection{Quantitative Results}

The flow states for the full-length duct are plotted in the $A-\theta$ phase plane in figure 5, where $A \equiv \Delta \rho /(2 \rho+\Delta \rho)$ is the Atwood number, a nondimensional measure of the initial density difference between the reservoirs (Charru 2011). These states were determined from visualisations and are somewhat subjective. Consequently, there is imprecision in the exact locations of the transitions from one state to another. As described above there are transitions from the $\mathrm{L}$, through the $\mathrm{H}, \mathrm{I}$ and $\mathrm{T}$ states as $A$ and $\theta$ increase. The $\mathrm{L}$ state is restricted to $\theta<0$, which corresponds to the along-duct component of gravity retarding the flow driven by the buoyancy-induced pressure differences across the ends of the duct. For $\theta=-1^{\circ}$, this retarding force is sufficient to laminarise the flow even for quite significant density differences $A \sim 0.1$. As $\theta$ increases the $\mathrm{H}$ state sets in for decreasing $A$ and was present even for the smallest $A \sim 10^{-3}$ for $\theta \geqslant-0.25^{\circ}$.

In many, but not all experiments, the net mass flux averaged over an experiment was determined by measuring, as described in $\S 2$, the change in the mean densities of the two reservoirs between the start and the end of an experiment. Assuming that there are constant, equal (and opposite) volume fluxes $Q$ along the duct over the duration $\tau$ of an experiment, conservation of mass for each reservoir implies that

$$
Q \tau=V \frac{\Delta A_{\mathrm{L}}}{A}=-V \frac{\Delta A_{\mathrm{R}}}{A},
$$

where $\Delta A$ is the change in the reservoir Atwood number over the time $\tau$ and the subscript $\mathrm{L}(\mathrm{R})$ implies the left (right) reservoir both of which have (equal) volume $V$. Thus from the independent reservoir density measurements we can estimate the volume flux $Q$ and from this, in turn, determine whether there has been any significant barotropic flow during the initial phase of the experiment. On average the two measures of $Q$ differed by less than $10 \%$, showing that barotropic effects were small. Experiments, where the difference in the two values of $Q$ differed by more than $10 \%$, were rejected from the analysis of the mass exchange throughout the duct. 


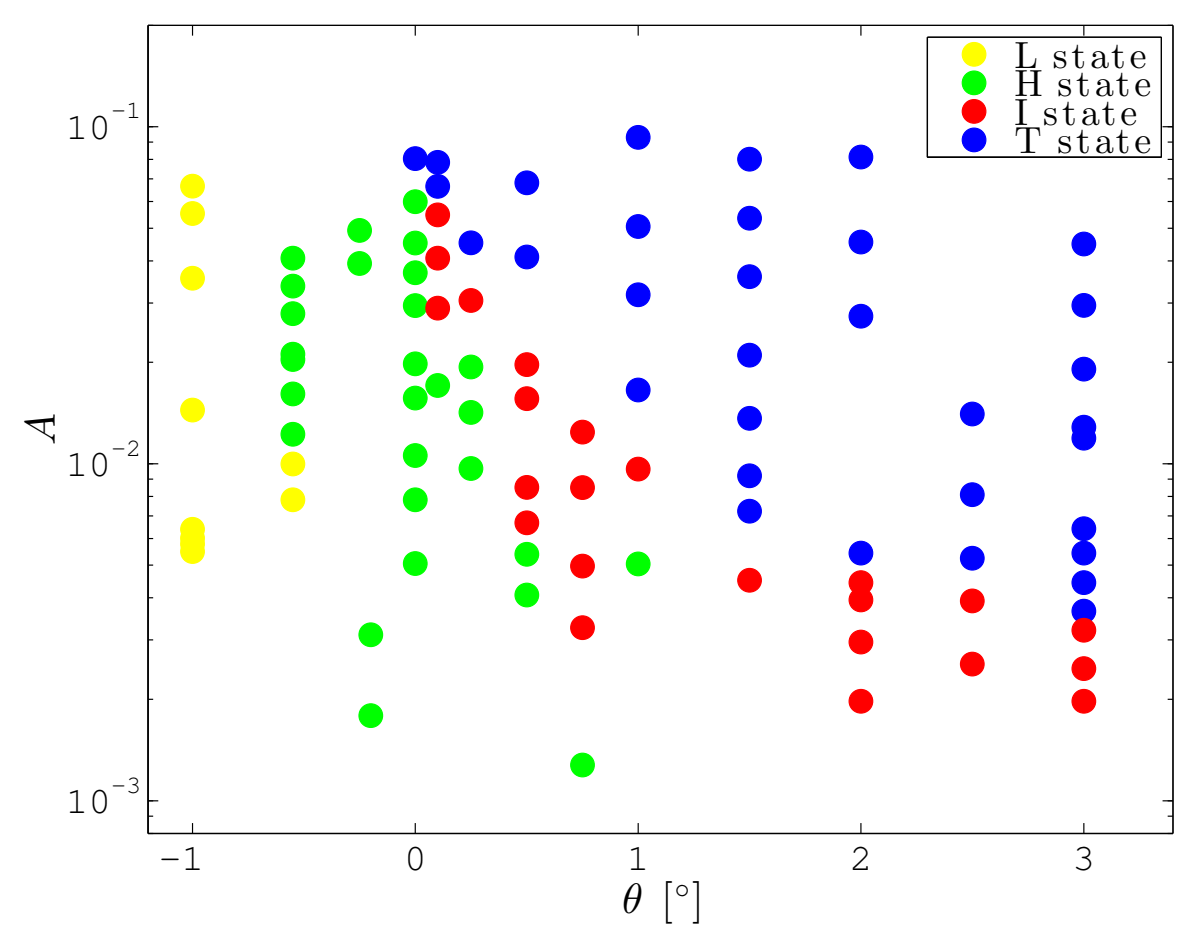

FIGURE 5. State space of the observed flows as a function of the duct inclination angle $\theta$ and the Atwood number $A$. These data are for the full $3 \mathrm{~m}$ duct.

From these measurements we can determine time-averaged 'mass flux' layer velocities $U=$ $2 Q / S$, where $S$ is the cross sectional area of the duct. In the absence of mixing between the layers this velocity is a measure of the layer-average velocity. In the presence of significant mixing between the two layers the salt flux will be reduced while the volume flux will not. Hence the layer-average velocity will be larger, and the mass-flux velocity provides a lower bound to the actual layer-average velocities. This average 'mass-flux' velocity $U$ is plotted against $\sqrt{g A H}$ in figure 6. The data for each individual duct inclination $\theta$ fall on straight lines indicating that the speeds scale with $\sqrt{g A H}$. Note that these results scale over the full range of Atwood numbers, including the highest values $A \sim 0.1,\left(\sqrt{g A H} \sim 0.30 \mathrm{~m} \mathrm{~s}^{-2}\right)$ which correspond to the largest density differences $\left(\Delta \rho \sim 200 \mathrm{~kg} \mathrm{~m}^{-3}\right)$, at which non-Boussinesq effects are largest. This suggests, as our visual observations of the phase states (figure 5) also show, that there are no significant qualitative or quantitative non-Boussinesq effects.

The dependence of velocity on the duct inclination is more clearly shown by plotting the layer Froude numbers $F \equiv U / \sqrt{g A H}$ as a function of $\theta$ (figure 7). These data include measurements from both the full duct and the half duct and cover the full range of flow states. At each angle the spread of values reflects the differences between flow states and the errors associated with the mass balances in the two reservoirs. There is, however, no apparent correlation with Atwood number and the average values (indicated by the white stars and diamonds for the full and half ducts, respectively) are consistent with one another, indicating little dependence on the duct aspect ratio. Further, these average values increase with increasing $\theta$ and then appear to asymptote to a value $F=0.61 \pm 0.05$ for $\theta \geqslant 1^{\circ}$. 


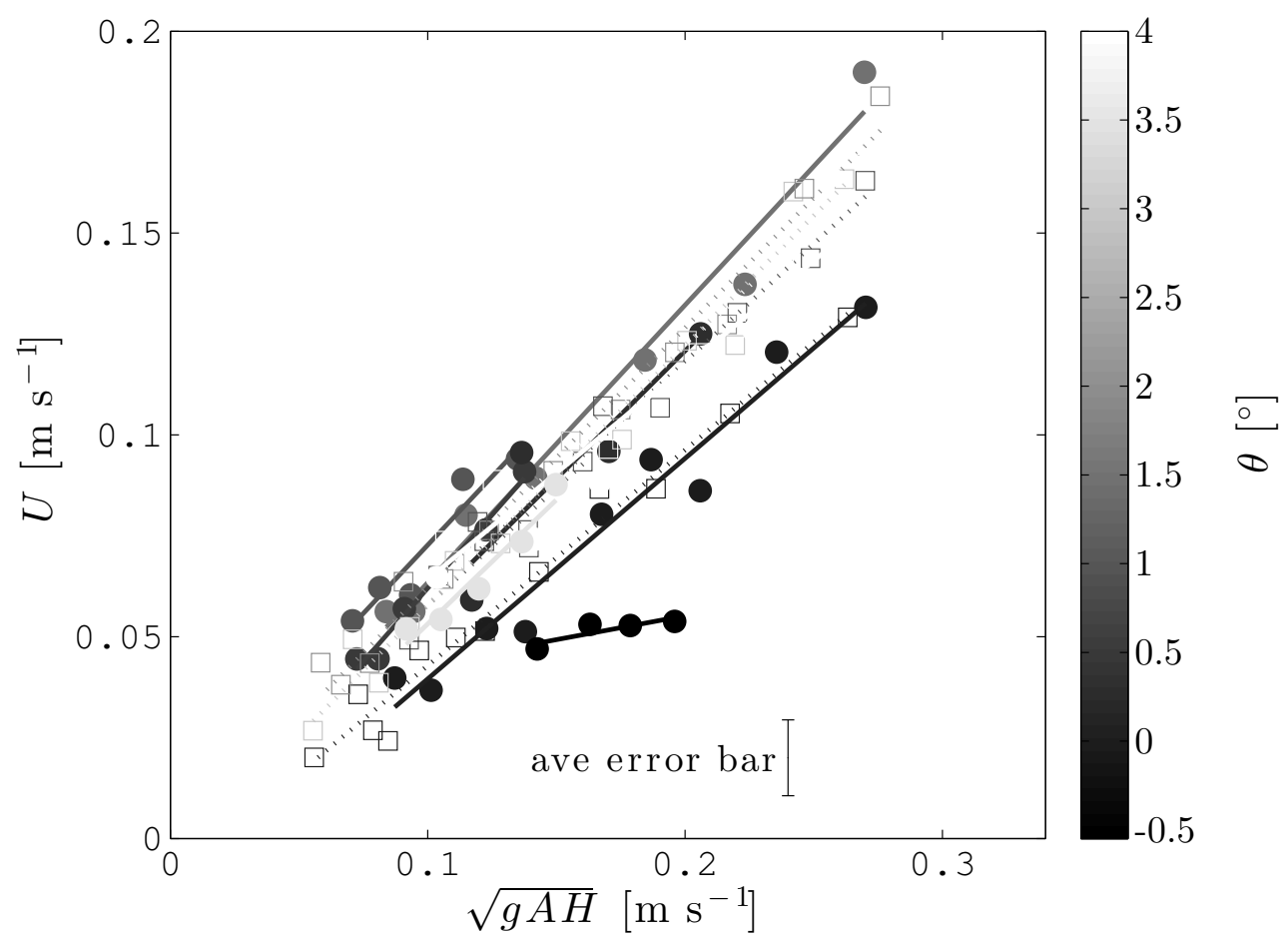

FIGURE 6. The averaged mass-flux velocity $U$ calculated from the change in densities of the two reservoirs over the duration of an experiment plotted against $\sqrt{g A H}$. The lines join experiments with the same inclination, indicated by the grey scale. Closed circles are for the full duct and open squares indicate the half duct. The error bar is an average over all experiments and is a result of the discrepancies between the mass balances for the two reservoirs.

\section{Force balances}

The flow states are determined by the density difference between the ends of the duct, the duct aspect ratio $H / L$ and the angle of inclination $\theta$. Assuming that the flow is steady and Boussinesq (an assumption supported by our measurements and observations described above), the alongduct momentum equation is

$$
u \frac{\partial u}{\partial x}=-\frac{1}{\rho_{0}} \frac{\partial p}{\partial x}+2 g A \sin \theta+\epsilon
$$

where $u$ is the along-duct velocity, $p$ is the pressure, $g$ is the gravitational acceleration and $\epsilon$ represents dissipative terms. Here we consider the dissipation term $\epsilon$ to represent different physical processes in the different states. In the $L$ state it represents viscous friction on the duct walls and across the interface, while in the other states it is associated with the different flow structures e.g. Holmboe modes and turbulence.

As noted above the $\mathrm{L}$ state is only observed when $\theta<0$ and the term $g A \sin \theta$ provides a deceleration to the flow. As shown in figure 2, the flow appears to be hydraulically controlled at both ends of the duct and there is no evidence of hydraulic jumps within the duct itself. If the connection between the reservoirs had been a slowly varying contraction there would be a single 


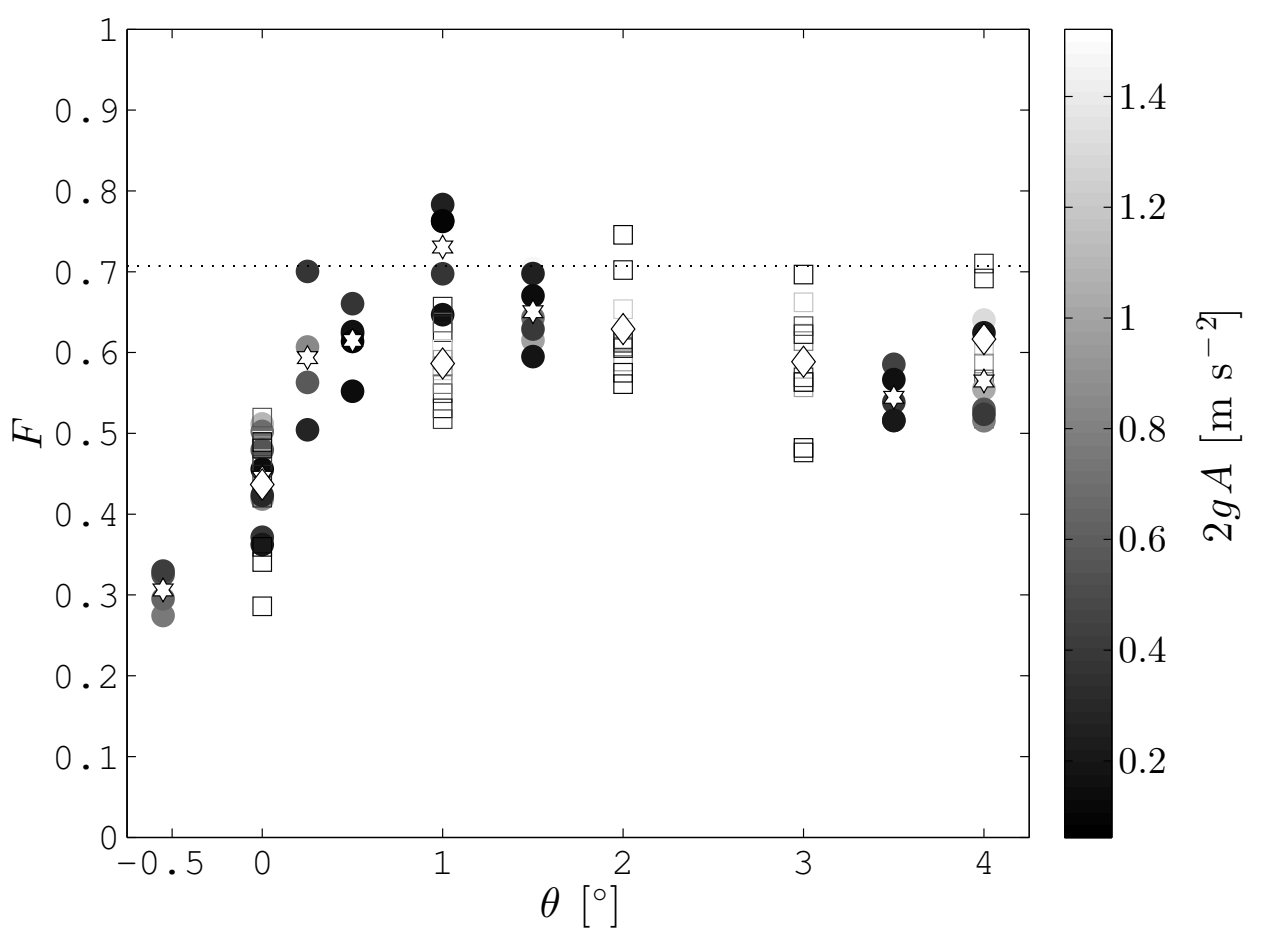

FIGURE 7. Froude numbers for each layer calculated from the mass flux velocities plotted against $\theta$. The white stars and diamonds are the average for each value of $\theta$ for the full and half ducts respectively. The dotted line is $F=1 / \sqrt{2}$. Closed circles are for the full duct and open squares indicate the half duct.

control point at which the composite Froude number $G$ satisfies

$$
G \equiv \sqrt{F_{1}^{2}+F_{2}^{2}}=1
$$

where $F_{i}=U_{i} / \sqrt{2 g A H_{i}}, i=1,2$ are the Froude numbers for the two layers (Armi 1986; Dalziel 1991). Thus for equal and opposite flows this implies that the Froude number for each layer is $F=1 / \sqrt{2}$. In the case of the duct with its abrupt entrances and exits, the control splits into two, one at each end of the duct, and there is a transition from critical to supercritical flow in the exiting layer (figure 2). Between these two controls the flow in the duct remains critical and, in the absence of dissipation, is governed by equation (4.2) (Tedford et al. 2009). However, as shown by figures 3 and 5 the flow is in either the $\mathrm{H}$ or I states with non-zero dissipation associated with Holmboe wave breaking or other turbulent mechanics. Consequently, the measured values at $\theta=0^{\circ}$ of $F=0.48 \pm 0.05$ are less than the dissipationless limit (0.71) but consistent with hydraulic theory including dissipation, and also consistent with horizontal duct measurements of Gu \& Lawrence (2005) who found in a shorter duct, with a larger cross-section and an upper free surface, a value of $F=0.55$.

In the horizontal case the flow is driven by the pressure gradient associated with the density difference at the ends of the duct. When the pressure is the same at each end at the height of the mid plane of the duct and is hydrostatic in the reservoirs, the average pressure difference between the ends of the duct for both the upper and the lower layer is $\rho_{0} g A / 2$. Hence the alongslope component of gravity becomes comparable with this horizontal pressure gradient when 


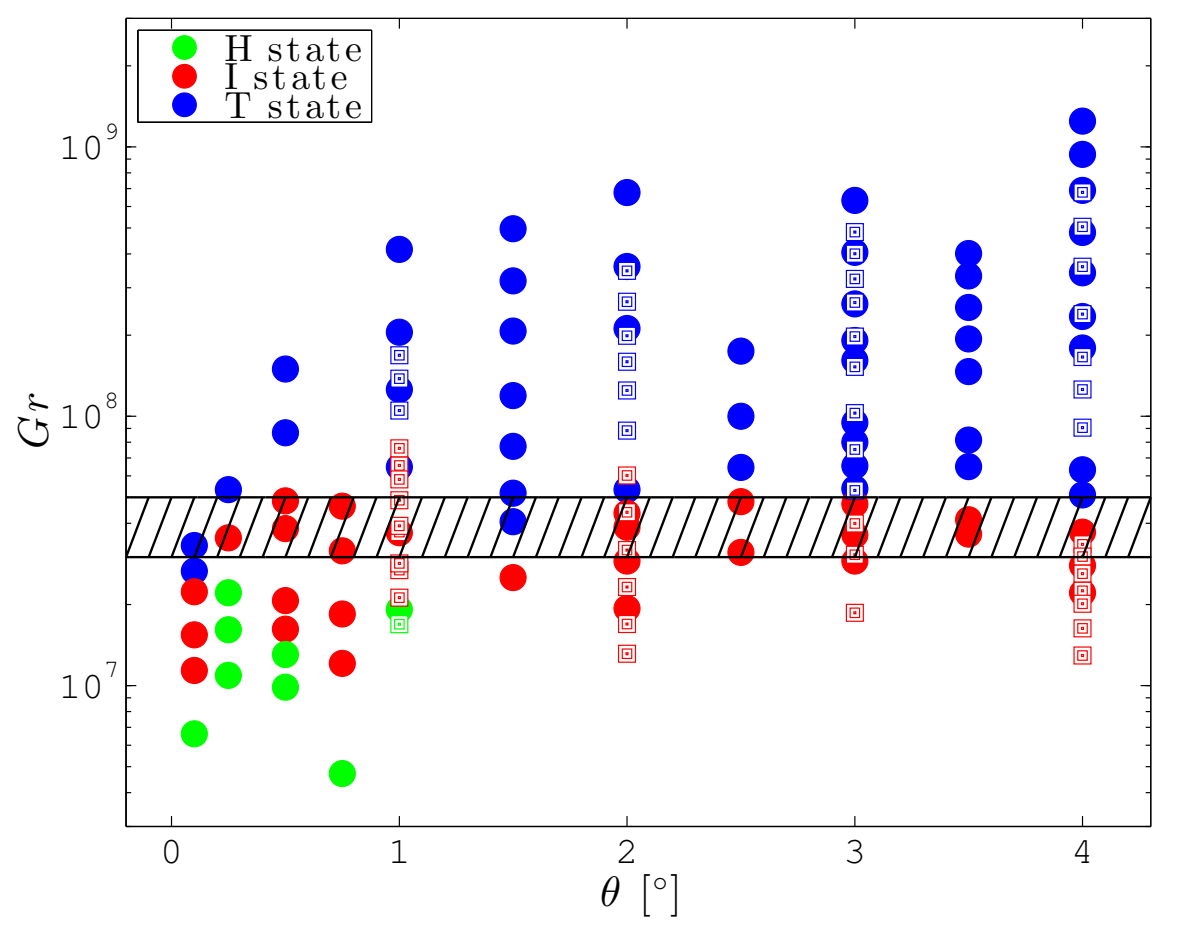

FIGURE 8 . The scaled state-space for $\theta \geqslant 0$ : Grashof number $g A L \sin \theta H^{2} / \nu^{2}$ vs $\theta$. Data include both the full length $(3 \mathrm{~m})$ duct (closed circles) and the half-length $(1.5 \mathrm{~m})$ duct (open squares). The transition to the T state scales independent of the duct length $L$.

$$
\frac{g A H}{2 L} \sim 2 g A \sin \theta
$$

Thus the along-slope acceleration becomes a significant driving factor when $\sin \theta \sim H / 4 L=$ $1 / 120(1 / 60)$ or $\theta \geqslant 0.5^{\circ}\left(1^{\circ}\right)$ for the full (half) duct. This is consistent with the observed increase in $F$ over the range to about $\theta=1^{\circ}$ (figure 7). For larger values of $\theta$ the pressure difference associated with the difference in height of the two ends of the duct is larger than that associated with the density difference in the reservoirs.

At these larger values of $\theta$ the predominant force balance is now between the along-slope component of buoyancy and dissipation. In the absence of dissipation this gravitational force produces a constant acceleration on each layer (Thorpe 1968) implying that $U \sim 2 g A t \sin \theta$. Over the time it takes for fluid to transit the duct $t \sim L / U$ this acceleration would produce a layer kinetic energy (per unit mass) $U^{2} \sim g A L \sin \theta$. The observations (figure 7) show on the other hand that $U^{2} \sim g A H$ and so the excess kinetic energy $U^{2}=g A \sin \theta(L-H) \approx g A L \sin \theta$, since $H \ll L$.

Consequently, for $\theta \gtrsim 1^{\circ}$, in order to maintain the observed velocities, the flow must dissipate this excess kinetic energy and we may postulate this occurs through a transition to a highly dissipative turbulent state. Thus we expect the transition to scale on a critical value of $g A L \sin \theta$, and we can express this transition non-dimensionally in terms of a critical Grashof number

$$
G r \equiv \frac{g A L \sin \theta H^{2}}{\nu^{2}} .
$$


Figure 8 shows data for the full-length $(3 \mathrm{~m})$ duct and experiments with a half-length $(1.5 \mathrm{~m})$ duct for $0.125^{\circ} \leqslant \theta \leqslant 4^{\circ}$. In the evaluation of $G r$ we have taken the value of the kinematic viscosity $\nu$ to be the average of that of the two reservoir salt solutions. We observe that the transition to the $\mathrm{T}$ state occurs around $G r \sim 4 \times 10^{7}$, for both the full-length and the halflength ducts. There is some uncertainty about the exact numerical value since the transition to the $\mathrm{T}$ state was made from qualitative observations. The hatched region in figure 8 is intended to reflect this uncertainty. The intermittent I states are characterized by spatio-temporal fluctuations in the turbulent intensity and regions with little dissipation. Image snapshots over time, such as figure 4, show such temporal fluctuations. For the T state, highly dissipative regions consistently develop and persist with no significant change of form throughout an experiment (figure 3(d)). Furthermore, the $\mathrm{T}$ states show structures spanning the interface from one side to the other and are characterised by a predominance of small scales, consistent with high levels of dissipation.

\section{Conclusions}

We present new experimental observations of the flow in an inclined duct separating two reservoirs at different densities. We observe four different flow states transitioning from a laminar $\mathrm{L}$ state at negative angles when the along-slope component of gravity decelerates the flow, through a wave-dominated $\mathrm{H}$ state, an intermittent I state, and finally a turbulent $\mathrm{T}$ state as the angle and the density difference increase. We show that the transitions between these states are a result of changing dynamics from a hydraulically controlled low dissipation state for a horizontal duct, to a high-dissipation state at large positive angles and density differences.

As mentioned in $\S 1$, where our range of angles overlap, Kiel (1991) observed similar flows to those reported here. However, he did not provide the values of the density differences so it is not possible to make direct comparisons with our state diagram (figure 5), and he writes that the 'shape of the density profiles was independent of $g^{\prime}(=2 g A$ in our notation)'. Kiel (1991) identified four flow regimes, one labeled 'stable counterflow' which corresponds to the L-state, and three other regimes that we would identify as T-states, and apart from observations at $\theta=0^{\circ}$, correspond to larger inclination angles. These latter three regimes are characterised by the increasing magnitude of the thickness of the interface region compared to the duct height. Tedford et al. (2011) note that Holmboe modes are observed when their duct is horizontal, and when it is tilted $(\theta>0)$, the 'shear increases and Kelvin-Helmholtz instabilities are generated. The instabilities break down the sharp interface between the two-layers resulting in a broad region of mixing. Subsequent leveling of the tube results in a three-layer exchange flow.' Again these observations are consistent with ours.

The velocities, estimated by mass-flux balances, at a given angle scale on the driving pressure difference $\sqrt{g A H}$ and the resulting layer Froude numbers $F$ are functions only of $\theta$, with $F$ increasing with increasing angle up to $\theta \approx 1^{\circ}$ and then remaining approximately constant with further increase in $\theta$. We note that the asymptotic value of $F \sim 0.6$ at large $\theta$ is consistent with the flow remaining hydraulically controlled at the ends of the duct. The along-slope gravitational acceleration is balanced by increased dissipation in the $T$ state and the resulting layer velocities are then given approximately by the two-layer hydraulic theory, in which the acceleration and the dissipation balance.

We found that the transition from the I state to the $T$ state occurred around a critical value of the Grashof number $G r \sim 4 \times 10^{7}$. In terms of the Reynolds number $R e=\sqrt{G r}$, this implies a critical value $R e \sim 6,000$, which is consistent with the 'mixing transition' value of $\sim 10^{4}$ identified by Dimotakis (2005). Additionally, we note that recent scaling (Brethouwer et al. 2007; Bartello \& Tobias 2013) has suggested that the buoyancy Reynolds number, based on local horizontal scales $l$ and vertical scales $h$, and given by $\mathscr{R} \equiv R e h^{2} / l^{2}$, is a more relevant parameter when the flow is stratified since vertical scales are suppressed compared with horizontal scales. 
It is difficult ascertain these scales from our current measurements, but from the shadowgraph images of the T state (figure 3(d)) it appears that the structures in the interface have aspect ratios $h / l \sim 0.1$ (as an order of magnitude estimate) in which case the critical value of $\mathscr{R} \sim$ 100. Bartello \& Tobias (2013) show from numerical calculations that $\mathscr{R}>\mathrm{O}(10)$ is required for viscous effects to be unimportant in strongly stratified flows. In contrast to many previous experiments on stratified turbulence such as those examining decaying turbulence, the present experiment allows these high values of the buoyancy Reynolds number to be achieved and maintained.

\section{Acknowledgements}

We would like to thank Stuart Dalziel and Graham Hughes for helpful discussions and Simon Vincent for running the experiments on the half-length duct. This work was supported by an EPSRC Programme Grant EP/K034529/1 and CRM was supported by a Churchill Scholarship.

\section{REFERENCES}

ARMI, L. 1986 The hydraulics of two flowing layers with different densities. J. Fluid Mech. 163, 27-58.

Bartello, P \& Tobias, S 2013 Sensitivity of stratified turbulence to the buoyancy Reynolds number. $J$. Fluid Mech. 725, 1-22.

Brethouwer, G., Billant, P., Lindborg, E. \& Chomaz, J.-M. 2007 Scaling analysis and simulation of strongly stratified turbulent flows. J. Fluid Mech. 585, 343-368.

CAUlfield, C. P. 1994 Multiple linear instability of layered stratified shear flow. J. Fluid Mech. 258, 255-285.

Charru, F. Hydrodynamic Instabilities. Cambridge University Press, 2011.

DALZIEL, SB 1991 Two-layer hydraulics: a functional approach. J. Fluid Mech. 223, 135-163.

Dimotakis, P. E. 2005 Turbulent mixing. Ann. Rev. Fluid Mech. 37, 329-356.

Gu, L \& LAwrence, G. A. 2005 Analytical solution for maximal frictional two-layer exchange flow. $J$. Fluid Mech. 543, 1-17.

Holmboe, J. 1962 On the behaviour of symmetric waves in stratified shear flow. Geofys. Publ. 24, 67-113.

KIEL, D. E. 1991 Buoyancy driven counterflow and interfacial mixing. PhD thesis, Cambridge.

Lawrence, G. A., Browand, F. K. \& Redekop, L. G. 1991 The stability of a sheared density interface. Phys. Fluids A 3, 2360-2370.

REYNOLDS, O. 1883 An experimental investigation of the circumstances which determine whether the motion of water shall be direct or sinuous, and of the law of resistance in parallel channels. Proceedings of the Royal Society of London 35 (224-226), 84-99.

Tedford, E. W., Carpenter, J., Pieters, R. \& Lawrence, G. A. 2011 Instabilities in a titling channel with open ends. In 7th Int. Symp. on Stratified Flows. Rome.

Tedford, E. W., Pieters, R. \& LAwrence, G. A. 2009 Symmetric Holmboe instabilities in a laboratory exchange flow. J. Fluid Mech. 636, 137-153.

THORPE, S. A. 1968 A method of obtaining a shear flow in a stratified fluid. J. Fluid Mech. 32, 693-704.

THORPe, S. A. 1971 Experiments on the instability of stratified shear flows: miscible fluids. J. Fluid Mech. 46, 299-319.

THORPe, S. A. 1973 Experiments on instability and turbulence in a stratified shear flow. J. Fluid Mech. $61(04), 731$. 